\title{
Preliminary data on precision of QuantiFERON-TB Plus performance
}

\author{
To the Editor:
}

We welcome the findings by BARCELLINI et al. [1] in their recent publication on the first independent evaluation of QuantiFERON-TB Plus (QFT-Plus; QIAGEN GmbH, Hilden, Germany). The authors conclude that the addition of novel peptides, aimed at stimulating a $\mathrm{CD}^{+} \mathrm{T}$-cell response, resulted in a diagnostic sensitivity for culture-confirmed tuberculosis of $88 \%$ suggesting that the QFT-Plus might offer improved sensitivity when compared with the most recent meta-analysis for QuantiFERON-TB Gold In-Tube (QFT-GIT) [2]. The findings also reinforce recent evidence supporting the theory that $\mathrm{CD} 8^{+} \mathrm{T}$-cells have a major role in host defence by stimulating the production of interferon- $\gamma$ and other soluble factors, activating macrophages which in turn suppress the growth of Mycobacterium tuberculosis (MTB) [3]. We agree with the statement of BARCELLINI et al. [1] on the need to corroborate such findings and assess sensitivity in $\mathrm{CD} 4^{+} \mathrm{T}$-cell depleted patient populations, as in HIV-infected patients where it has been shown that $\mathrm{CD}^{+} \mathrm{T}$-cells associate with active tuberculosis (TB) [4].

While recognising the importance of providing further insight into the best use of an interferon- $\gamma$ release assay (IGRA) related $\mathrm{CD}^{+}$signal in defining the test's role in programmatic TB infection management [5], we feel that test reproducibility ought also to be assessed. Within subject IGRA variability has been reported in recent research $[6,7]$. Such variability could affect test reproducibility and possibly explain the occurrence of apparent conversion/reversion phenomena, particularly in serial testing of low-risk individuals such as healthcare workers.

To assess any potential change in precision of the novel test, as part of an in-house validation exercise, we compared within subject variability between the QFT-Plus and QFT-GIT. As part of the validation of the QFT-GIT, we collected five samples from two donors (10 samples in total) of known MTB status (subject 1: positive and subject 2: negative) via direct collection of blood into the relevant tubes (nil, antigen and mitogen). We sampled the same two subjects for in-house validation of the new QFT-Plus and collected 10 samples from each subject (20 samples in total) into the relevant tubes (nil, TB1, TB2 and mitogen). Samples were well mixed and incubated at $37^{\circ} \mathrm{C}$ for $16-24 \mathrm{~h}$, then centrifuged at $2500 \times g$ for 15 min. Two separate Grifols Triturus automated ELISA analysers (Grifols UK Ltd, Cambridge, UK) (referred to as Triturus A and B) were used to perform the assay sequence at room temperature. The data produced were analysed using QFT-GIT analysis software V2.62 and QFT-Plus analysis software V2.70.

All results for both assays were either positive or negative and no indeterminate results were demonstrated. In both the QFT-GIT and the QFT-Plus, the data for subject 1 expressed $100 \%$ positive results indicating likely MTB infection, whereas subject 2 demonstrated 100\% negative results indicating no MTB infection. The coefficient of variation (CV) of each batch of subjects on each analyser was calculated based on the mean value for TB antigen/TB1/TB2 minus the nil. With the QFT-GIT, subject 1 expressed a CV for TB antigen-nil of 17.27 and 19.22 on Triturus A and B, respectively, whereas with the QFT-Plus the CVs of TB1/TB2-nil were $10.75 / 8.10$ and $10.98 / 8.55$ on Triturus A and B, respectively. Subject 2, however, produced no reaction therefore there was little variation in the CV of TB antigen/TB1/TB2 minus the nil, with both the QFT-GIT ELISA and the QFT-Plus ELISA expressing a CV of 0.00-0.01 (figure 1).

The objective of this study was to compare the precision of the new QFT-Plus with the QFT-GIT. From the data obtained, qualitatively there is no variation between the two assays; however, when looking at the in-batch data, a lower CV for the QFT-Plus is detected where there has been a positive reaction, as observed in subject 1.

The data obtained from the new QFT-Plus ELISA gave a mean CV of $9.60 \%$ in comparison with a mean CV of $18.25 \%$ for the QFT-GIT ELISA. The reduction in CV could be particularly important for results around the cut-off point of $0.35 \mathrm{IU} \cdot \mathrm{mL}^{-1}$, by reducing the chance of finding of reversion/conversion phenomena in low-risk individuals where low prevalence decreases the positive predictive value of the test. This research has been conducted within a small target population as an in-house confirmation of the research produced by the manufacturer [8] and presented in the product package insert. While this is useful for in-house validations, larger studies are needed with enough statistical power to allow full assessment of the new assay's precision in the context of QFT-Plus result variability. This, along with assessment of sensitivity and predictive value for incident TB, will provide the necessary body of evidence 

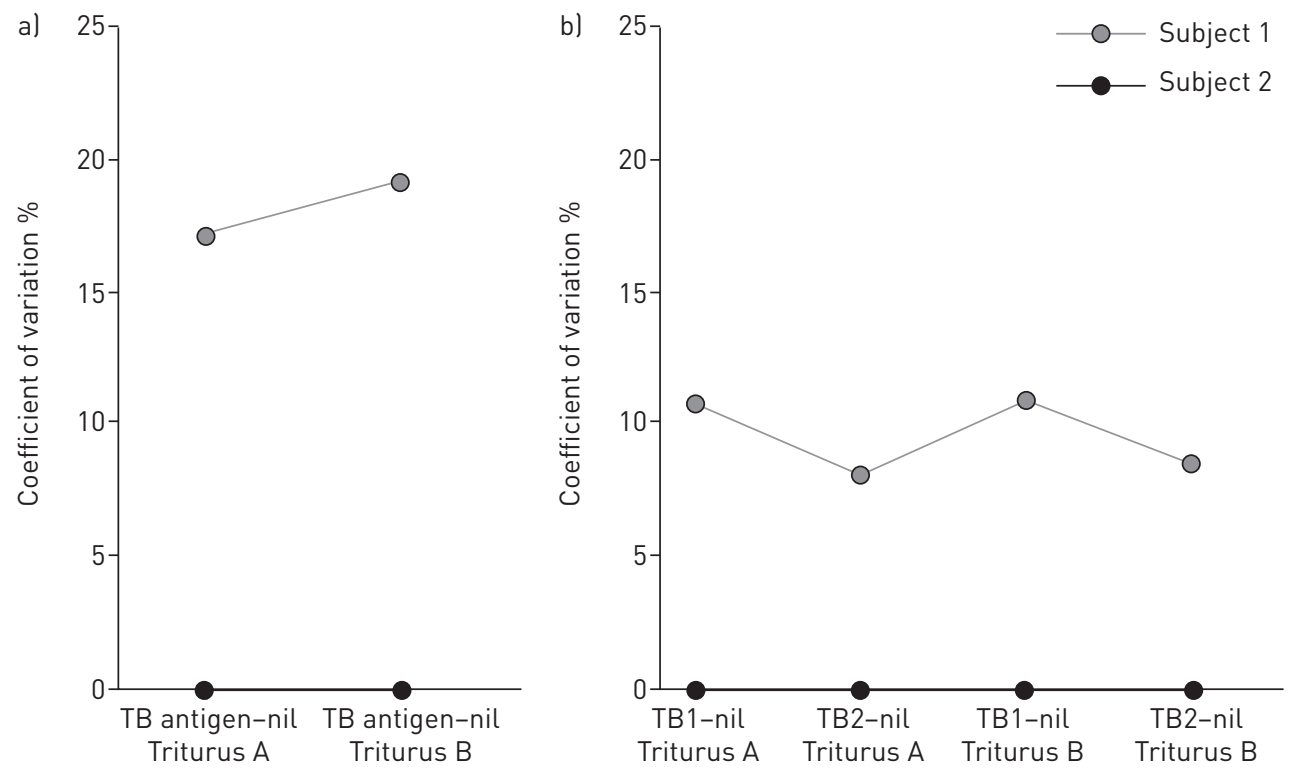

FIGURE 1 Coefficient of variation based on average antigen/TB1/TB2-nil for a) the QuantiFERON-TB Gold In-Tube (QFT-GIT) and b) QuantiFERON-TB Plus (QFT-Plus).

to guide clinical and programmatic use of QFT-Plus within the context of TB infection management and TB elimination policy $[9,10]$.

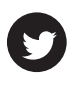

@ERSpublications

QFT Plus variability of $\mathbf{9 . 6 \%} v s \mathbf{1 8 . 2 5 \%}$ in QFT Gold: this could reduce TB reversion/conversion in low-risk individuals http://ow.ly/W97W300mUtZ

David Gallagher $^{1}$, Davide Manissero ${ }^{2}$, Christopher Stocking ${ }^{1}$ and Chris Pyne ${ }^{1}$

${ }^{1}$ Synlab UK Ltd, London, UK. ${ }^{2}$ QIAGEN Manchester Ltd, Manchester, UK.

Correspondence: David Gallagher, Synlab UK Ltd, C/O HMR, 44 Cumberland Avenue, London, NW10 7EW, UK.

E-mail: David.Gallagher@synlab.com

Received: March 232016 | Accepted after revision: April 292016

Support statement: QIAGEN GmbH, Hilden, Germany provided the QuantiFERON-TB Plus kits free of charge.

Conflict of interest: Disclosures can be found alongside this article at erj.ersjournals.com

\section{References}

1 Barcellini L, Borroni E, Brown J, et al. First independent evaluation of QuantiFERON-TB Plus performance. Eur Respir J 2016; 47: 1587-1590.

2 Sester M, Sotgiu G, Lange C, et al. Interferon- $\gamma$ release assays for the diagnosis of active tuberculosis: a systematic review and meta-analysis. Eur Respir J 2011; 37: 100-111.

3 Prezzemolo T, Guggino G, La Manna MP, et al. Functional signatures of human CD4 and CD8 T cell responses to Mycobacterium tuberculosis. Front Immunol 2014; 5: 180.

4 Chiacchio T, Petruccioli E, Vanini V, et al. Polyfunctional T-cells and effector memory phenotype are associated with active TB in HIV-infected patients. I Infect 2014; 69: 533-545.

5 Getahun H, Matteelli A, Abubakar I. Management of latent Mycobacterium tuberculosis infection: WHO guidelines for low tuberculosis burden countries. Eur Respir J 2015; 46: 1563-1576.

6 van Zyl-Smit RN, Zwerling A, Dheda K, et al. Within-subject variability of interferon-g assay results for tuberculosis and boosting effect of tuberculin skin testing: a systematic review. PLoS One 2009; 4: e8517.

7 Tagmouti S, Slater M, Benedetti A, et al. Reproducibility of interferon gamma (IFN- $\gamma$ ) release assays: a systematic review. Ann Am Thorac Soc 2014; 11: 1267-1276.

8 QIAGEN. QuantiFERON ${ }^{-}$-TB Gold Plus (QFT'-Plus) ELISA Package Insert. www.quantiferon.com/irm/content/ PI/QFT/PLUS/2PK-Elisa/UK.pdf Date last accessed: March 13, 2015. Date last updated: February 2015.

9 Diel R, Loddenkemper R, Zellweger J-P, et al. Old ideas to innovate TB control: preventive treatment to achieve elimination. Eur Respir J 2013; 42: 785-801.

10 Lönnroth K, Migliori GB, Abubakar I. Towards tuberculosis elimination: an action framework for low-incidence countries. Eur Respir J 2015; 45: 928-952. 